

http://www.TIMCS.com

The Journal of Mathematics and Computer Science Vol .1 No.4 (2010) 293-304

\title{
Simulating a state of the art agent based system with fuzzy reasoning for supply chain coordination
}

\author{
Ali Shemshadi1, ${ }^{*}$, Hossein Shirazi ${ }^{2}$, M. J. Tarokh ${ }^{3}$ \\ K. N. Toosi University of Tech, shemshadi@sina.kntu.ac.ir \\ Malek Ashtar University of Tech, shirazi@mut.ac.ir \\ K. N. Toosi University of Tech,mj.tarokh@kntu.ac.ir
}

Received: September 2010, Revised: November 2010

Online Publication: December 2010

\begin{abstract}
With the emergence of high speed wireless networks and parallel advancements in Internet based technologies such as the Semantic Web, Web Services, Multi Agent Systems, and Context Awareness, the realization of the vision of the intelligent wireless web (IWW) has become a possibility. On the other side, recent developments in the field of multi criteria decision making, have led us to more accurate and applicable algorithms. In this paper, we are going to summarize the details and results of a multidisciplinary agent based software, namely SWESS that enables real-time supply chain coordination through taking advantage of different methods of different scientific areas such as computer science, supply chain management and multi criteria decision making.
\end{abstract}

Keywords: Supply chain coordination, supply chain management, multi-criteria decision making, multi agent systems, artificial intelligence

\section{Introduction}

\footnotetext{
$1{ }^{*}$ Corresponding author: Postgraduate student at the department of information technology \& industrial engineering, K. N. Toosi University of Technology, Tehran, Iran.

${ }^{2}$ Associate Professor at the department of information and communication technology, Malek Ashtar University of Technology, Tehran, Iran.

${ }^{3}$ Associate Professor at the department of information technology \& industrial engineering, K. N. Toosi University of Technology, Tehran, Iran.
} 
Coordination is considered as the management of dependencies between activities. [1] The purpose of coordination is to achieve collectively goals that individually cannot be met. Effectively integrating the information and material flows within the demand and supply process is the main concern for Supply Chain Management (SCM). The potential for improved productivity, cost reduction, and customer service are enormous [2].

According to [3] effective supply chain coordination and integration, as one of the main critical areas of SCM, is one of the major challenges to have a successful implementation of mobile SCM. Based on supply chain's decision making structure and the nature of demand, different coordination mechanisms might be taken to integrate supply chain's activities. [4] Recent trends towards the convergence of wireless communications and Internet based technologies have the potential to open new avenues of mobile collaboration, thereby minimizing the impact of the physical dispersion of supply chain members. Intelligent Wireless Web integrates artificial intelligence and wireless technology into the World Wide Web in order to make it more powerful and more tuned to the real needs of the user [6].

Section 2 of this paper starts by inquiring into related works and the vision of the intelligent wireless web based services. Then other related areas of research, including mobile real-time supply chain coordination, are reviewed. The third section investigates more deeply into the technical and architectural specifications of the simulated software. Section 4 concludes the paper.

\section{Related works}

In this section we will review basic concepts and methods that were deployed to implement SWESS.

\subsection{Intelligent Wireless Web}

\subsubsection{Building Blocks}

IWW is defined as a "network that provides anytime, anywhere access to information resources with efficient user interfaces and applications that learn and thereby provide increasingly useful services whenever and wherever needed" [7]. The idea of IWW goes beyond merely connecting mobile devices to the Internet. It encompasses the creation of a pervasive, user centered mobile work environment, which has the ability to provide highly specific data and services to users on as-needed basis (i.e. in real-time), by intelligent interpretation of the user context. Table 1 illustrates the key building blocks of the IWW.

Table 1- The building blocks of IWW

\begin{tabular}{|c|l|l|}
\hline & \multicolumn{1}{|c|}{ Building Block } & \multicolumn{1}{c|}{ Role } \\
\hline 1 & Existing Desktop Based Infrastructure & Separation of data from presentation and applications \\
\hline 2 & High Bandwidth Wireless Technologies & Provide the vital communication between the wired back-end and the wireless front-end \\
\hline 3 & Semantic Intelligence & Enabling knowledge description (using ontology) and knowledge access \\
\hline 4 & Web Services & Ensure dynamic discovery of resources and resource integration \\
\hline 5 & Agents & $\begin{array}{l}\text { Plays the key role in addressing issues such as security, negotiation, personalization and } \\
\text { procurement }\end{array}$ \\
\hline
\end{tabular}

\subsection{Mobile Real-time Supply Chain Coordination}

This section takes a step in the direction of introducing the critical role of mobile real-time coordination in the supply chain. A supply chain comprises interdependent units within a firm and across units that belong to both downstream and upstream partners [9]. Given the nature of the interdependence between units, mobile real-time coordination is a necessary prerequisite to integrate their operations to achieve the mutual goal of the supply chain as a whole as well as those of its units [10].

Real-time coordination of activities between units becomes important as firms focus on their core activities and outsource the rest - thus their success increasingly depends on their ability to coordinate their internal and external activities in the value chain outside their own boundaries on an as needed basis [11].

Effective coordination between units in a supply chain has come to play a key role in focusing on the innovation, flexibility, and speed that serve as the sources of competitive advantage necessary for survival in global competition [12]. 
Different industries and businesses are currently struggling to implement techniques that can reduce supply process duration and cost, whilst improving productivity and performance, and doing these all in a mobile real-time manner. In this regard, there has been a movement towards the use of collaborative processes that leverage the Internet platform, thereby allowing participation of the supply chain members, from the very early stages of the supply process. However, these collaborative working solutions are tailored primarily towards the needs of desktop-based fixed network client. The collaboration needs of site-based staff and other mobile people are not well addressed [7].

\subsubsection{Coordination Mechanism}

According to the classifications done by [13], we can categorize supply chain coordination mechanisms into three categories:

- Price Coordination: Quantity discount pricing schemes are among the most frequently studied mechanisms in the price coordination area

- Non-price Coordination: Non-price coordination mechanisms include quantity flexibility contracts, allocation rules, promotional allowances, cooperative advertising, and exclusive dealings/territories

- Flow Coordination: Flow coordination mechanisms are designed to manage product and information flows in supply chains.

An IWW enabled MRSCC mainly improves coordination mechanisms in the Flow Coordination Mechanisms area, which can be listed as [13]:

- Quick Response

- Collaborative Planning, Forecasting and Replenishment

- Efficient Consumer Response

To make the proposed system practical there also should be incentives for supply chain members. Here are some of them:

- With the sudden changes of material and stuff prices in today's business environment, many supply chain members wish to get free of long-term contracts. Participating in an IWW enabled mobile real-time supply chain makes this wish true.

- With the growing size and complexity of today and near future's supply chains, there are many cases that the presence of human beings or traditional systems to operate the supply chain operations becomes impossible or too costly. MIT's Space Net project [14] and such projects would be a thorough example for these supply chains.

- Through running an ERP, it would be possible for supply chain members to use this system to make their procurements either.

\section{Simulation Results}

\subsection{Reference Architecture}

To illustrate the synergy between the various technologies discussed, a practical deployment scenario is presented. This scenario illustrates the case of a mobile supply chain member, who is in this case a worker, generating a real-time purchase order for a standard construction component. The following numbers illustrate the chronology of interaction, and correspond to the numbers in Figure 1. 


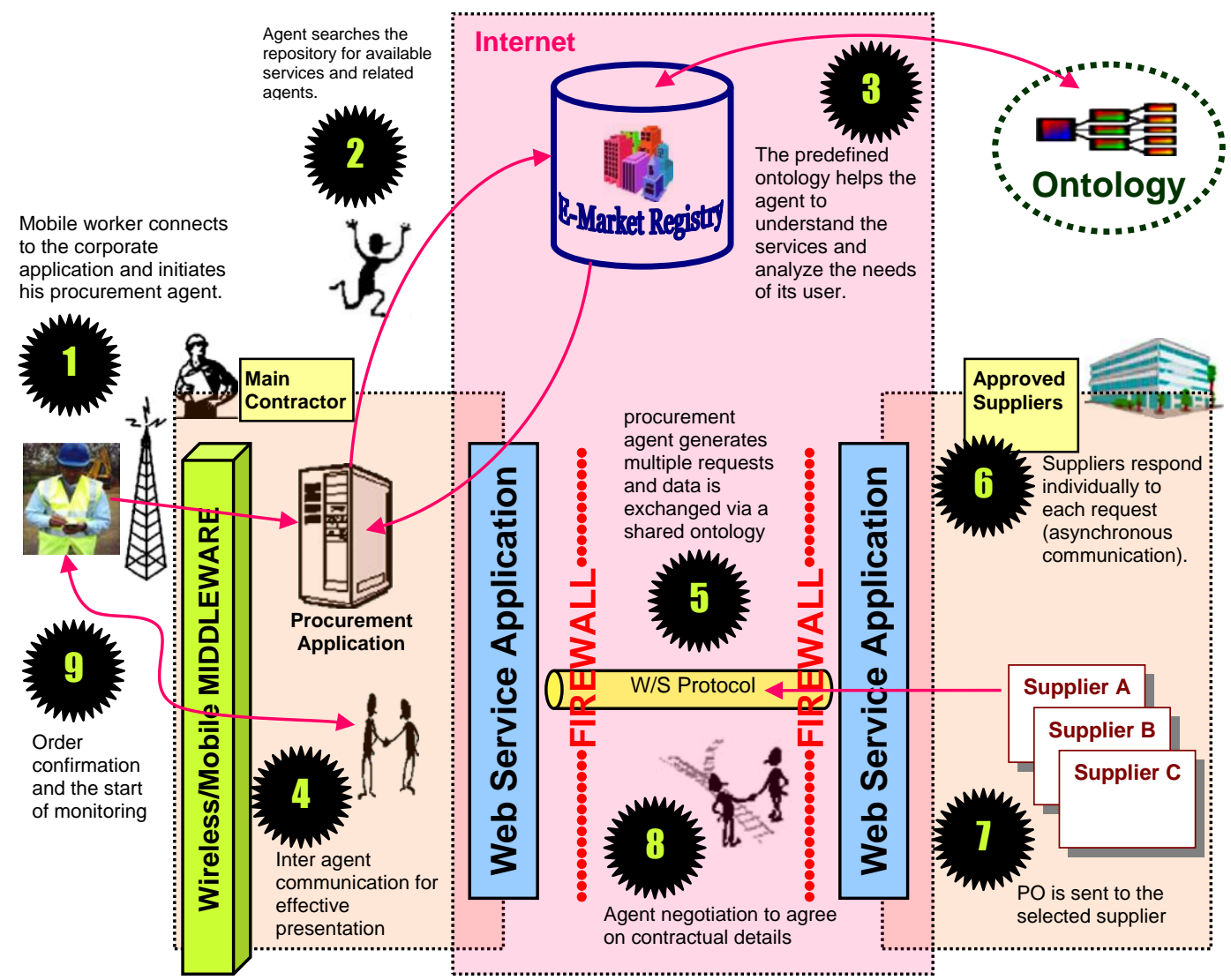

Figure 1- Standard component supply scenarios in a mobile real-time supply chain coordination system[8] 
following technologies were deployed to implement the software:

Java Development Kit (JDK) 1.6, Java Agent Development Framework 3.7, Microsoft SQL Server 2008 Enterprise Edition, Protégé, XML and AJAX.

Some snapshots of the implemented software have been shown in Figures 2 and 3.

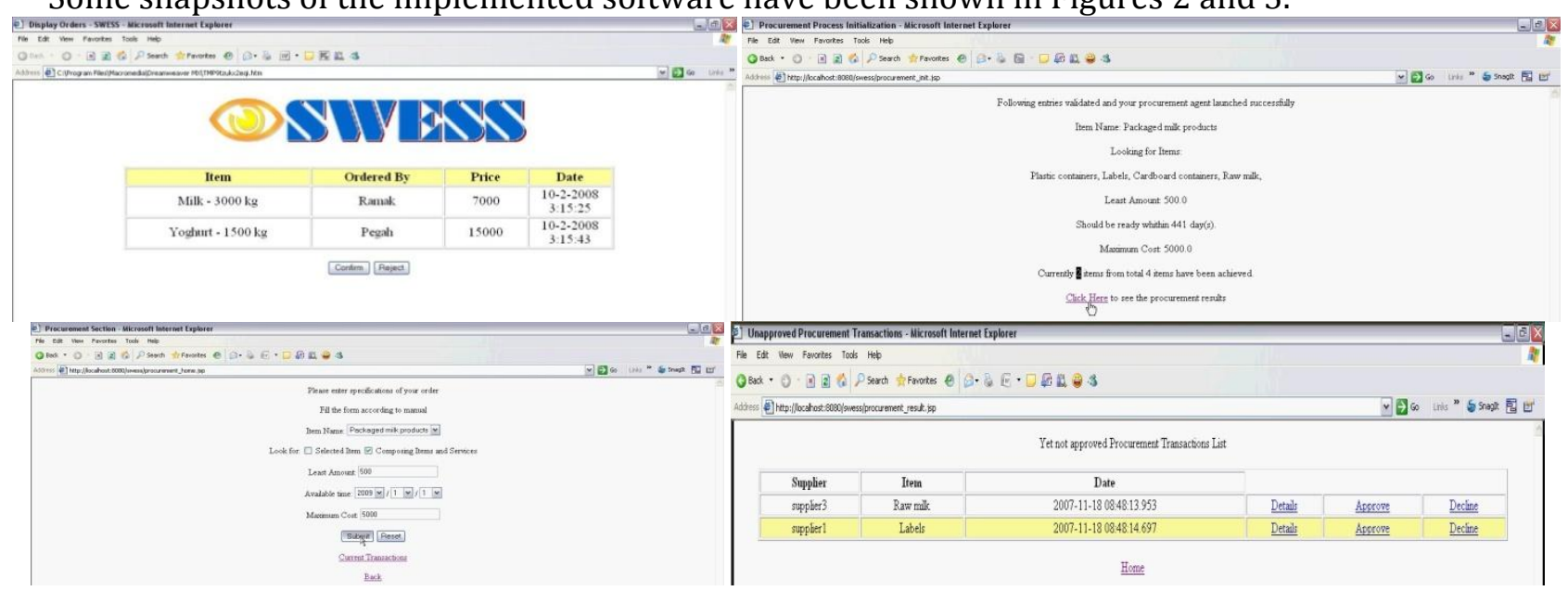

Figure 2- Snapshots of SWESS's web based user interface

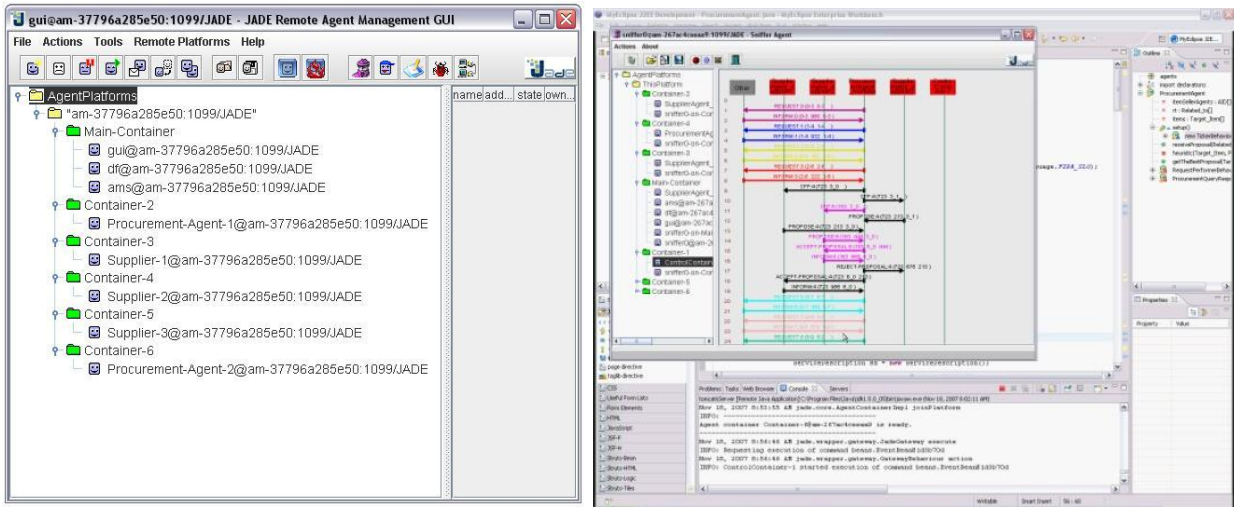

Figure 3- Snapshot of JADE's RMA GUI during running SWESS

\subsection{MAS architecture}

To make JADE's agents accessible through JSP pages; a new agent named gateway agent, should be added to our multi agent system. Thus we used ACL messages inside MAS and use bean objects named board beans outside the MAS.

\subsection{Agent negotiations \& procurement mechanism}

In SWESS, negotiation and purchasing is done with a certain mechanism. Figures 4 and 5 illustrate this mechanism. Its steps could be enumerated as followings:

Startup, creating agent, adding new items, looking for suppliers, sending CFP, Proposing item's cost, choosing item with the best price, finalizing the purchase, saving transaction into database, getting buy results, getting sale results, and terminating agents.

SWESS should provide a simple interface for its users (both suppliers and buyers).

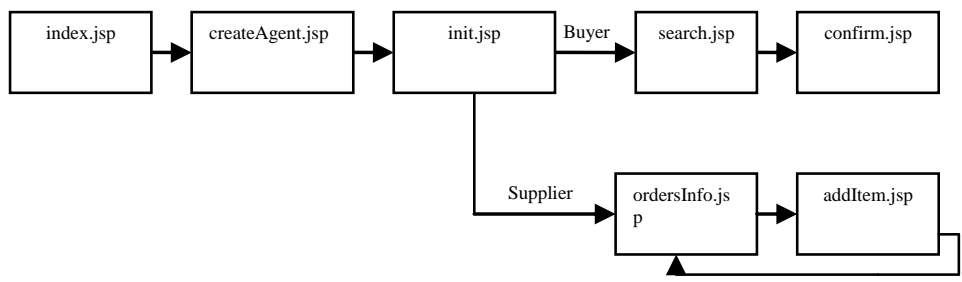

Figure 4- Procurement steps in SWESS 
Ali Shemshadi, Hossein Shirazi, M. J. Tarokh/ TJMCS Vol .1 No.4 (2010) 293-304

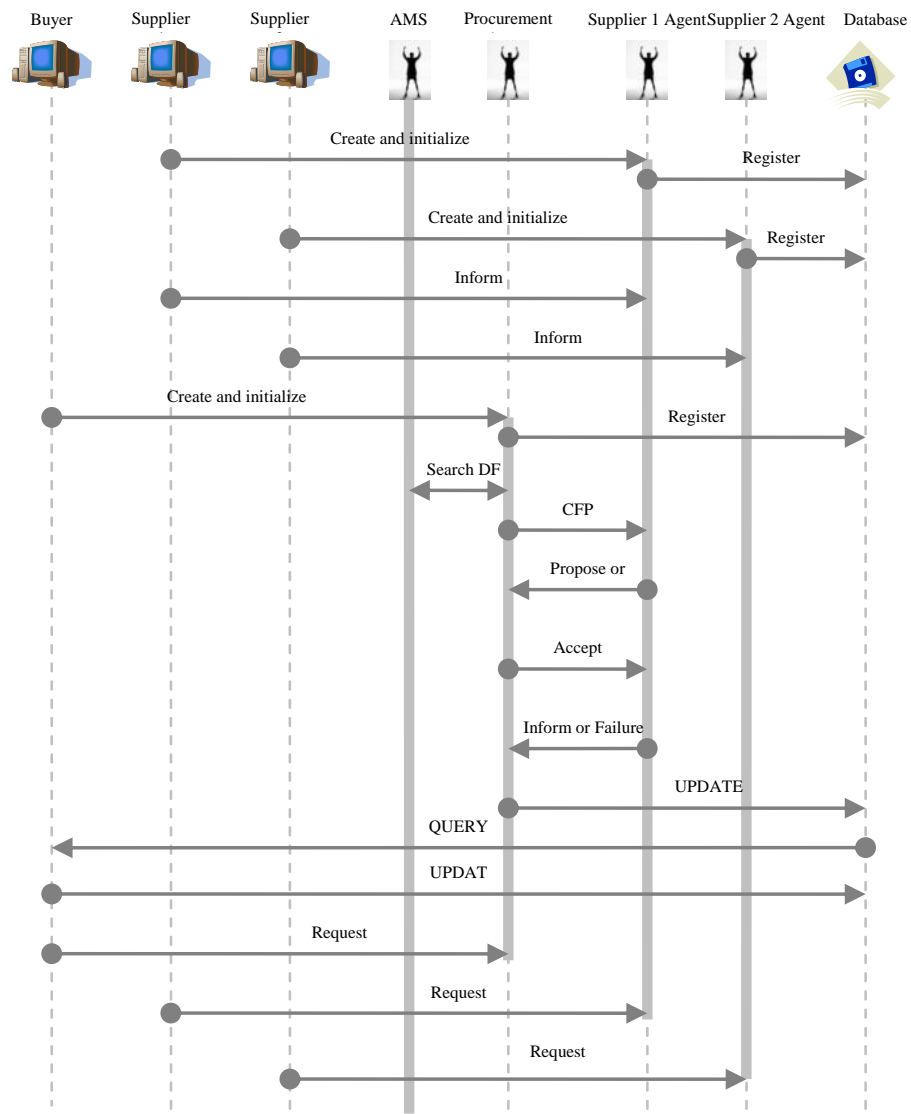

\subsection{Performance analysis}

Figure 5- Illustration of SWESS's Negotiation and Purchasing Mechanism.

Let's discuss about SWESS's running cost analysis. With the subsequent assumptions:

- $c_{s}$ : cost of launching and initializing a new agent

- $c_{d}$ : cost of database access by agents

- $c_{m}$ : cost of one message transport

- $c_{t}$ : cost of agent termination

Let's suppose that $P$ procurement agents and $S$ supplier agents should run and procurement agent $i$, is looking for $N_{i}$ items at all, where item $\mathrm{j}$ is available by $S_{j} \leq S$ suppliers. Thus the cost for procurement agent i will be:

$C_{i}=c_{s}+c_{d}+2 c_{m}+N_{i} \times\left(\sum_{k=1}^{S_{j}}\left(2 c_{m}\right)+3 c_{m}\right)+c_{d}+c_{t}$

$=c_{s}+2 c_{d}+2 c_{m}+c_{t}+N_{i} \times\left(\sum_{k=1}^{s_{j}}\left(2 c_{m}\right)+3 c_{m}\right)$

Because in practical cases supplier agents start or terminate rarely, we can ignore its cost, therefore cost of running the system for all agents equals to:

$$
\begin{aligned}
& C=\sum_{i=1}^{P} C_{i}=\sum_{i=1}^{P}\left[c_{s}+2 c_{d}+2 c_{m}+c_{t}+N_{i} \times\left(\sum_{k=1}^{S_{j}}\left(2 c_{m}\right)+3 c_{m}\right)\right] \\
& =P \times\left(c_{s}+2 c_{d}+2 c_{m}+c_{t}\right)+\sum_{i=1}^{P}\left[N_{i} \times\left(\sum_{k=1}^{S_{j}}\left(2 c_{m}\right)+3 c_{m}\right)\right]
\end{aligned}
$$




\subsection{Technical details}

3.5.1. Agent Communication Protocols.

Agents constitute the foundation of the proposed manufacturing control system; thus, in order to observe the critical standards for information systems analysis and design, and to implement the agents communication mechanism based on globally approved notations and rules, we employed the latest standards for developing multi-agent modules. The applied agent communication protocols are [17]: FIPA ACL Protocol, FIPA Contract Net Protocol, FIPA Request When Interaction Protocol, and FIPA SL Protocol.

\subsubsection{Ontology.}

Ontology (the "science of being") is a word that is used in many different senses. It is sometimes considered to be identical to metaphysics, but we prefer to use it in a more specific sense, as that part of metaphysics that specifies the most fundamental categories of existence, the elementary substances or structures out of which the world is made. Recently, the term of "ontology" has been up taken by researchers in Artificial Intelligence, who use it to designate the building blocks out of which models of the world are made [18].

An agent, such as an autonomous robot using a particular model, will only be able to perceive that part of the world that his ontology is able to represent. In a sense, only the things in his ontology can exist for that agent. In that way, ontology becomes the basic level of a knowledge representation scheme [19].

Regarding our multi-agent modules for the distributed manufacturing control system, we use a knowledge-base to implement the pertaining ontology of these agents. To develop the ontology, we comply with the ontology designed by Protégé [20] which is compatible with OWL [21]. Another research is under development to offer the ontology model for this system.

\subsubsection{Implementation Environment.}

The proposed distributed manufacturing control system is being implemented using Java Agent DEvelopment framework (JADE). This multi-agent platform is conformant with the standard specifications of the Foundation for Intelligent Physical Agents (FIPA). These specifications are a collection of standards, which are intended to promote the interoperation of heterogeneous agents. This compliance guarantees the interoperability of JADE agents and other agents, which comply with the same standard [22].

\subsection{Supplier Selection Method}

In this section we are going to propose an extended version of VIKOR that supports subjective and objective weights. The problem of supplier selection in supply chain system could be treated as a group multiple criteria decision making (GMCDM) problem, which could be described by means of following sets (Chena et al., 2006):

1. A set of $m$ possible alternatives called $A=\left\{A_{1}, A_{2}, \ldots, A_{m}\right\}$;

2. A set of $n$ decision criteria called $C=\left\{C_{1}, C_{2}, \ldots, C_{n}\right\}$;

3. A set of $K$ decision makers called $D=\left\{D_{1}, D_{2}, \ldots, D_{k}\right\}$;

4. A set of utility ratings of $A_{i}(i=1,2, \ldots, m)$ with respect to criteria $C_{j}(j=1,2, \ldots, n)$ called $X=\left\{x_{i, j} / i=1,2, \ldots m ; j=\right.$ $1,2, \ldots, n\}$.

The main steps of the proposed supplier selection algorithm could be described as followings:

Step 1: Identify and define linguistic terms and relevant membership functions.

Two set of appropriate linguistic variables are needed to estimate the importance weight of each criterion (figure 3 ) and the fuzzy rates of alternatives assigned by decision makers (Figure 4).

Step 2: Construct a decision matrix.

Let the fuzzy rating for $i$ th alternative regarding $j$ th criterion of $k$ th decision maker be shown as $x_{i j k}=\left(x_{i j k 1}, x_{i j k 2}, x_{i j k 3}\right)$ and the importance weight of the $j$ th criterion given by the $k$ th decision maker be $w_{j k}^{s}=\left(w_{j k 1}^{s}, w_{j k 2}^{s}, w_{j k 3}^{s}\right)$. Hence, the aggregated fuzzy rating $\left(\tilde{x}_{i j k}\right)$ with respect to criterion $C_{j}$ could be calculated as:

$x_{i j k}=\left\{\left(x_{i j k 1}, x_{i j k 2}, x_{i j k 3}\right) \mid i=1,2, \ldots, m ; j=1,2, . ., n ; k=1,2, \ldots, K\right\}$ and 


$$
\begin{aligned}
& \left\{\begin{array}{l}
x_{i j 1}=\min _{k}\left\{x_{i j k 1}\right\} \\
x_{i j 2}=\frac{1}{K} \sum_{k=1}^{K} x_{i j k 2} \\
x_{i j 3}=\max _{k}\left\{x_{i j k 3}\right\}
\end{array}\right. \\
& \left\{\begin{array}{l}
w_{j 1}^{s}=\min _{k}\left\{w_{j k 1}^{s}\right\} \\
w_{j 2}^{s}=\frac{1}{K} \sum_{k=1}^{K} w_{j k 2}^{s} \\
w_{j 3}^{s}=\max _{k}\left\{w_{j k 3}^{s}\right\}
\end{array}\right.
\end{aligned}
$$$$
\text { Similarly, the aggregated subjective importance weights }\left(\tilde{w}_{j}^{s}\right) \text { could be calculated }
$$$$
\text { using the following relations: }
$$$$
w_{j}^{s}=\left\{\left(w_{j 1}^{s}, w_{j 2}^{s}, w_{j 3}^{s}\right) \mid j=1,2, . ., n\right\} \text { and }
$$

Thus, the decision matrix $\widetilde{D}$ and the subjective weighting matrix $\widetilde{W}^{s}$ could be concisely expressed as:

$$
\begin{aligned}
\tilde{D} & =\left[\begin{array}{cccc}
x_{11} & x_{12} & \ldots & x_{1 n} \\
x_{21} & x_{22} & \ldots & x_{2 n} \\
: & : & : & : \\
x_{m 1} & x_{m 2} & \ldots & x_{m n}
\end{array}\right] \\
\sim & \tilde{\sim} \\
W^{s} & =\left[\begin{array}{llll}
\tilde{w}_{1}^{s} & \tilde{w}_{2}^{s} & \ldots & \tilde{w}_{n}^{s}
\end{array}\right]
\end{aligned}
$$

Step 3: Defuzzify the decision matrix and fuzzy weights of each criterion and derive their crisp values.

To derive the crisp values of arrays of decision matrix and fuzzy weights we may use the following equations:

$$
\begin{aligned}
\operatorname{defuzz}\left(x_{i j}\right) & =\frac{\int \mu(x) \cdot x d x}{\int \mu(x) d x} \\
& =\frac{-x_{i j 1} x_{i j 2}+x_{i j 2} x_{i j 3}+\frac{1}{3}\left(x_{i j 3}-x_{i j 2}\right)^{2}-\frac{1}{3}\left(x_{i j 2}-x_{i j 1}\right)^{2}}{-x_{i j 1}+x_{i j 3}}
\end{aligned}
$$

Step 4: Deploy the entropy concept to derive objective weights.

In order to determine the objective weights by entropy measure, first we should normalize the decision matrix for each criterion $C_{j}(j=1,2, \ldots, \mathrm{n})$ and calculate the projection value of each criterion called $P_{i j}$.

$$
P_{i j}=\frac{x_{i j}}{\sum_{i=1}^{m} x_{i j}}
$$

Afterward, the entropy value can be calculated as follows:

$e_{j}=-k \sum_{j=1}^{n} P_{i j} \ln \left(P_{i j}\right)=-\frac{1}{\ln (m)} \sum_{j=1}^{n} P_{i j} \ln \left(P_{i j}\right)$

Then, to calculate the degree of divergence $d i v_{j}$ of the intrinsic information of each criterion $C_{j}(j=1,2, \ldots, \mathrm{n})$ we may deploy the following equation

$d i v_{j}=1-e_{j}$

The value of $\operatorname{div}_{j}$ represents the inherent contrast intensity of each criterion $C_{j}$. The higher the $\operatorname{div}_{j}$ is, the more important criterion $C_{j}$ becomes for the problem. The objective weight for each criterion $C_{j}$ can be calculated as

$w_{j}^{o}=\frac{d i v_{j}}{\sum_{j=1}^{n} d i v_{j}}$ 
Ali Shemshadi, Hossein Shirazi, M. J. Tarokh/ TJMCS Vol .1 No.4 (2010) 293-304

Step 5: Obtain the decision matrix to identify the $j$ th criterion with respect to $i$ th alternative and normalize it in order each criterion value is limited between 0 and 1 , so the criterions will be comparable.

$$
\begin{aligned}
& U=\left[u_{i j}\right]_{m \times n} \\
& x_{i j 3}^{+}=\max _{i}\left\{x_{i j 3}\right\}, C_{j} \in B \\
& x_{i j 1}^{-}=\min _{i}\left\{x_{i j 1}\right\}, C_{j} \in C \\
& u_{i j}=\left\{\begin{array}{l}
\left(\frac{x_{i j 1}}{x_{i j 4}^{+}}, \frac{x_{i j 2}}{x_{i j 4}^{+}}, \frac{x_{i j 3}}{x_{i j 4}^{+}}\right), C_{j} \in \text { Benefit } \\
\left(\frac{x_{i j 1}}{x_{i j 1}^{-}}, \frac{x_{i j 2}}{x_{i j 1}^{-}}, \frac{x_{i j 3}}{x_{i j 1}^{-}}\right), C_{j} \in \text { Cost }
\end{array}\right.
\end{aligned}
$$

Step 6: Calculate the overall performance evaluation.

$$
\begin{aligned}
F & =\left[f_{i j}\right]_{m \times n} \\
f_{i j} & =\operatorname{defuzz}\left(u_{i j} \otimes w_{j}^{s}\right) \\
f_{i j}= & \frac{\int \mu(x) \cdot x d x}{\int \mu(x) d x} \\
= & \frac{-\left(u_{i j 1} u_{i j 2}\right)\left(w_{j 1}^{s} w_{j 2}^{s}\right)+\left(u_{i j 2} u_{i j 3}\right)\left(w_{j 2}^{s} w_{j 3}^{s}\right)+\frac{1}{3}\left(u_{i j 3} w_{j 3}^{s}-u_{i j 2} w_{j 2}^{s}\right)^{2}-\frac{1}{3}\left(u_{i j 2} w_{j 2}^{s}-u_{i j 1} w_{j 1}^{s}\right)^{2}}{-u_{i j 1} w_{j 1}^{s}+u_{i j 3} w_{j 3}^{s}}
\end{aligned}
$$

Step 7: Determine the best $f_{j}^{*}$ and the worst $f_{j}^{-}$values of all criterion ratings.

$$
\begin{aligned}
& f_{i}^{*}=\max _{i}\left\{f_{i j}\right\} \\
& f_{i}^{-}=\min _{i}\left\{f_{i j}\right\}
\end{aligned}
$$

Step 8: Compute the values of $S_{i}$ and $R_{i}$ as follows:

$$
\begin{aligned}
& S_{i}=\sum_{j=1}^{n} \frac{w_{j}^{o}\left(f_{i}^{*}-f_{i j}\right)}{\left(f_{i}^{*}-f_{i}^{-}\right)} \\
& R_{i}=\max _{i}\left(\frac{w_{j}^{o}\left(f_{i}^{*}-f_{i j}\right)}{\left(f_{i}^{*}-f_{i}^{-}\right)}\right)
\end{aligned}
$$

Step 9: Compute the values $Q_{i}$ as follows:

$$
\begin{aligned}
& S^{-}=\max _{i}\left\{S_{i}\right\} \\
& S^{*}=\min _{i}\left\{S_{i}\right\} \\
& R^{-}=\max _{i}\left\{R_{i}\right\} \\
& R^{*}=\min _{i}\left\{R_{i}\right\} \\
& Q_{i}=\frac{v\left(S_{i}-S^{*}\right)}{S^{-}-S^{*}}+\frac{(1-v)\left(R_{i}-R^{*}\right)}{R^{-}-R^{*}}
\end{aligned}
$$


$v$ is introduced as a weight for the strategy of maximum group utility, whereas $1-v$ is the weight of the individual regret.

Step 10: Rank the alternatives sorting by values $S, R$ and $Q$ in an ascending order

Step 11: Propose as a compromise solution the alternative $(A(1))$ which is the best ranked by the measure $Q$ (minimum) if the following two conditions are satisfied:

1. C1. Acceptable advantage: $Q\left(A^{(2)}\right)-Q\left(A^{(1)}\right) \geq D Q$

Whereas $A^{(2)}$ with $2^{\text {nd }}$ position in the ranking list by $\mathrm{Q}$ and $D Q=1 / j-1$.

2. C2. Acceptable stability in decision making.

The alternative $A^{(1)}$ must also be the best ranked by $S$ or/and $R$. This compromise solution is stable within a decision making process, which could be the strategy of maximum group utility (when $v>$ 0.5 is needed), or "by consensus" $v \approx 0.5$, or "with veto" $v<0.5$. Here, $v$ is the weight of decision making strategy of maximum group utility.

If one of the conditions is not satisfied, then a set of compromise solutions is proposed, which consists of

- Alternatives $A^{(1)}$ and $A^{(m)}$ if only the condition C2 is not satisfied, or

- Alternatives $A^{(1)} ; A^{(2)} ; \ldots ; A^{(M)}$ if the condition C1 is not satisfied; $A^{(M)}$ is determined by the relation $Q\left(A^{(M)}\right)-Q\left(A^{(1)}\right) \geq D Q$ for maximum M (the positions of these alternatives are "in closeness").

\section{Conclusion}

The realization of the vision of the IWW is within reach (given the recent achievements in the enabling technologies) and will enhance the value proposition of mobile communications in mobile realtime coordination of supply chains. Delivering real-time, context relevant, and personalized information to mobile members of a supply chain will save valuable time and will improve efficiency and productivity. Also there is a need to integrate technology innovations in other areas, such as multimodal interfaces and speech technologies, to enhance the usability of the mobile devices.

However, a key challenge is to link various technological enabling elements such as those discussed in this paper with methodological, cultural, social, and organizational aspects specific to supply network processes and firms. This would require input from different scientific fields, including computer science, telecommunications, system design, and ergonomics.

This holistic multi-disciplinary approach is essential to address the relationship between component technologies, end users, business processes, and wireless technologies. To encourage widespread adoption, there is a need for successful industrial case studies of mobile real-time supply chain coordination. There are numerous potential benefits and the industries and businesses need to make the necessary investments to realize these.

This paper introduced an innovative, practical method to organize and develop the intelligent wireless web. Then, through a structured discussion, we went through an investigation of the technical and architectural details of the implementation of a mobile real-time supply chain coordination system via the IWW services.

The implemented software was tested for two different supply chains and the results can be summarized as followings:

- Supply chain of a diary: a standard and general supply chain scheme of a diary company was taken from [23] and [24]. The requirements of an applicable software in this case could be summarized as followings:

- Defining the exact and standard service descriptions for every member of a chain is necessary; 


\section{Ali Shemshadi, Hossein Shirazi, M. J. Tarokh/ TJMCS Vol .1 No.4 (2010) 293-304}

- Indicating each member's touch points with upstream and downstream members in chain is necessary.

- A shoe-making factory: The implemented software was and the deployed ontology system was tailored to be used in a local shoe factory. First, the supply chain coordination mechanism of factory was analyzed and the results were published in [25]. Then the software was tested and the results could be summarized as followings:

- Incentives for upstream and downstream members should be rethought previously;

- Applicability in both continuous activities and discrete projects could be useful;

- The need for a general and applicable monitoring framework is vital and undeniable;

- The security issues should be rethought and guaranteed.

- The decision making algorithm:

$\circ \quad$ The need for supporting group and individual decision making processes will lead us to more realistic applications;

- Applying more realistic methods as MCDM algorithms which continue to improve is profitable.

Each requirement indicated above, could be an useful field of research for future studies.

\section{References}

[1] T. Kaihara, "Multi-agent based supply chain modeling with dynamic environment", International Journal of Production Economics 85, pp. 263-269, 2003.

[2] M. J. Tarokh and J. Soroor, "Supply Chain Management Information Systems Critical Failure Factors", European Management \& Technology Conference of Technology Research Institute of Florida, Inc., Rome, Italy, 20-21 June 2005.

[3] Malone, T.W., Crowston, K. (1994), “The interdisciplinary study of coordination”, ACM Computer Surveys, Vol. 26, Iss. 1, pp.87-119.

[4] Xiuhui Li, Qinan Wang, "Coordination mechanisms of supply chain systems", European Journal of Operational Research, 2006.

[5] C. J. Anumba, Z. Aziz, and E. Obonyo, "Mobile Communications in Construction-Trends and Prospects, C. J. Anumba, "Innovative Developments in Architecture, Engineering and Construction", Millpress Science Publishers, Rotterdam, pp. 159-168, 2003

[6] H. P. Alesso and C. F. Smith, "The Intelligent Wireless Web", Addison Wesley, ISBN: 0201730634, 2002.

[7] Z. Aziz et al.، "Intelligent wireless web services for construction". Automation in Construction، 2005

[8] J. Soroor, M. J. Tarokh, and A. Shemshadi, "Initiating an State of the Art System for Real-time Supply Chain Coordination", European Journal of Operational Research, Vol. 196, Issue 2, 16 July 2009.

[9] T. M. Simatupang, I. V. Sandroto, and S. B. H. Lubis, "Supply chain coordination in a fashion firm", Supply Chain Management: An International Journal, Vol. 9, No. 3, pp. 256-268, 2004.

[10] T. M. Simatupang, A. C. Wright, and R. Sridharan, "The knowledge of coordination for supply chain integration", Business Process Management Journal, Vol. 8, No. 3, pp. 289-308, 2002.

[11] M. J. Tarokh and J. Soroor, "Using Enterprise Resource Planning Tools in Real time Supply Chain Coordination", 2nd International Conference on Information Management and Business, Sydney, Australia, February 2006.

[12] H. L. Lee, "Aligning supply chain strategies with product uncertainties", California Management Review, Vol. 44, No. 3, pp. 105-119, 2002.

[13] B. Fugate, F. Sahin, and J. T. Mentzer, "Supply Chain Coordination Mechanisms", Journal of business Logistics, 2006.

[14] http://spacelogistics.mit.edu.

[15] Saaty, T. L. (1999). Fundamentals of the Analytic Network Process. ISAHP Japan, pp. 12-14

[16] Saaty, T. L., (1996), Decision Making with Dependence and Feedback: The Analytic Network Process, RWS Publications, Pittsburgh.

[17] http://www.fipa.org/. 
Ali Shemshadi, Hossein Shirazi, M. J. Tarokh/ TJMCS Vol .1 No.4 (2010) 293-304

[18] H. Jagdev, "A semantic web service environment for B2B and B2C auction applications within extended and virtual enterprises", Computers in Industry 59, pp. 786-797, 2008.

[19] H.K. Lin and J.A. Harding, "A manufacturing system engineering ontology model on the semantic web for inter-enterprise collaboration", Computers in Industry 58, pp. 428-437, 2007.

[20] http://protege.stanford.edu/.

[21] http://www.w3.org/TR/owl-ref/.

[22] A. Shemshadi, J. Soroor, and M. J. Tarokh, "Implementing a Multi-Agent System for the Real-time Coordination of a Typical Supply Chain Based on the JADE Technology", Third IEEE SMC International Conference on System of Systems Engineering (SoSE'08), Portola Plaza Hotel, Monterey, California, USA, 2-4 June 2008.

[23] D. Reid, N. R. Sanders, "Operations Management", New York: John Wiley \& Sons, 2002, p. 80

[24] E. Turban, R. Kelly Rainer, R. E. Potter, "Introduction to Information Technology", $3^{\text {rd }}$ Edition, John Wiley \& Sons, 2004, p. 247-248

[25] Javad Soroor, M. J. Tarokh, Ali Shemshadi, "Theoretical and practical study of supply chain coordination", Journal of Business and Industrial Marketing (ISI-indexed), Emerald, Vol. 24, Issue 2, pp. 131-142, Feb. 2009. 